\title{
Spectrum Sensing Optimization by Sensing Threshold and Duration for Cognitive Radio Networks
}

\author{
Vinayak R. Barbole ${ }^{1}$, Prof. Anant R. More ${ }^{2}$, Prof. M.S.G. Prasad ${ }^{3}$ \\ Student(ME), Department of Electronics \& Telecommunication, RMD Sinhgad School of Engineering, Pune, India ${ }^{1}$ \\ Ph.D. Scholar, K.L. University, A.P. (India) \& Asst. Prof. RMD Sinhgad School of Engineering, Pune, India ${ }^{2}$ \\ Professor, Electronics and communication, KKR Institute of Technology \& Sciences, Guntur, India ${ }^{3}$
}

\begin{abstract}
The spectrum sensing problem is one of the challenging issues in cognitive radio systems. In this paper, Survey for Spectrum Sensing Optimization of cognitive radio networks by joint optimization of sensing Threshold and Duration is presented. High network utilization by secondary users can be achieved with fast and reliable spectrum sensing. Maximizing Throughput for secondary users by considering both parameter variable to sensing 1.Sensing Threshold and 2.Sensing Duration. Joint optimization can be achieved by two performance criteria minimizing time for detection of spectrum hole and Joint optimization of the opportunistic throughput.
\end{abstract}

Keywords: Cognitive radio, Joint optimization, Spectrum sensing, Sensing Threshold, Sensing Duration.

\section{INTRODUCTION}

A cognitive radio network (CRN) is a radio that can be into a bi-convex problem that allows ready computation of programmed and configured dynamically to use the the desired optimal values of key variables time. best wireless channels in its vicinity like a radio (integration time and threshold) automatically detect available channels in the wireless spectrum and then according to this changes it's transmission or reception parameters allow more concurrent wireless communications in a given spectrum band at one location. In a licenced band efficient spectrum sensing is critical when Primary and secondary users both present. In this case there may be miss-detection of active primary user or missing transmission even when channel is ideal. It creates two types of probabilities witch are the functions of both sensing threshold and duration Probability of false alarm and Probability of miss detection. In probability of false alarm channel is detected as ideal by secondary users even when primary user is present and In case of Probability of miss detection ideal band is detected as active.All proposed an optimal sensing duration in order to maximize achievable throughput of the secondary network.the authors extended thissingle frame optimization approach over multiple slots etc. All developed an optimization approach to maximize the aggregate throughput for optimal sensing thresholds for each sub-band. Increasing the detection threshold increases both the alarms false of primary users and successfully detection of spectrum holes. Similarly, integration over longer duration improves spectrum sensing performance for a given threshold of missdetections. Accordingly, we formulate an optimization problem of minimizing mean time to detection subject to constraints on the probabilities of issued detection and false alarm;Convert the resulting non-convex problems

\section{LITERATURE REVIEW}

With respect to said work an extensive literature survey is conducted accordingly which is presented as below,

1) Cognitive radio is being intensively researched for emergency opportunities access to the so-called TV White Spaces.(TVWS) huge portion of the VHF/UHF TV bands which become free on this basisof digital switchover. Using accurate digital TV (DTV) coverage maps together with a database of DTV transmitters, we developed a methodology for identifying TVWS frequencies at any given location in the United Kingdom. We implement our method to investigate the variations in TVWS as a function of location and transmit the power of cognitive radios and check how constraints on adjacent channel interference superimposed and regulators may effect on theresults.Ouranalysis provides a real view on the spectrum opportunity associated with cognitive devices, and presents the first quantitative study of availablefrequency composition of TVWS outside the United States.

2) The secondary users are allowed to use the frequency band of primary users in cognitive radio system when these bands are not currently being used. To support this spectrum reuse functionality, the secondary users are also required to sense the frequency environment, and once primary users are found to be active, the secondary users are required to vacate the channel within a certain amount 
of time thus the spectrum sensing is of significant importance in the cognitive radio networks. There are two parameters associated with spectrum sensing these are probability of detection and probability of false alarm. The higher the probability of detection; better the primary users are protected. However, In perspective of secondary users; the lower the probability of false alarm, more chances the channel can be reused when it is available. Thus higher the achievable throughput for the secondary network. In this paper, we study the problem of sensing the duration of maximize the achievable throughput ofsecondary network, under the primary users are sufficiently protected. We formulate the sensing throughput trade off problem mathematically, and use energy detection $\mathrm{s}$ scheme to prove that the formulated problem indeed has one optimal sensing time which provide the highest throughput for the secondary network. Cooperative sense using multiple mini slots or multiple secondary users can also studied using the methodology proposed.Computer simulations shown that for a $6 \mathrm{MHz}$ channel, when the frame duration is $100 \mathrm{~ms}$ andratio of signal to noise of primary user at the secondary receiver is $-20 \mathrm{~dB}$ the optimal sensing time achieving the highest throughput while maintaining $90 \%$ of probability of detection $14.2 \mathrm{~ms}$. This optimal sensing time decreases when distributed spectrum sensing is applied.

3) Spectrum sensing is an essential enabling functionality for cognitive radio networks to detect spectrum holes and opportunistically use the frequency bands without causing harmful interference to legacy networks. This paper introduce a novel wideband spectrum sensing technique called multi and joint detection, which jointly detects the signal energy levels over multiple frequency bands rather than considering one band at a time. The main strategy is effectively improvedthe dynamic spectrum utilization and reducing interference to the primary users. In the interference spectrum sensing problem is formulated as a class of optimization problems. By exploiting the hidden convexity in the seemingly non-convex problem formulations, optimal solutions for multiband joint detection are obtained under real time conditions. Simulation results shows that the proposed spectrum sensing scheme consistently improve the system performance.This article insicates the important principles for the design of wideband spectrum sensing algorithms in cognitive radio networks.

4) Traditionally, radios are constrained to operate within a band of frequencies that has been set aside for their sole use by regulatory bodies (so called licensed bands). But with many legacy technologies present and with many new wireless standards on the horizon, the sub $10 \mathrm{GHz}$ spectrum is quickly becoming saturated. Several known studies of spectrum utilization, however, definitively show that licensed and allocated spectrum is used desired effect than $50 \%$ of broadcast television channels in the Seattle, Washington area are unused, constituting known 'white spaces' in the spectrum. Dynamic spectrum access (DSA) has thus been proposed as a means to improve spectral efficiency by opening the spectrum for use by new secondary users on a non-interfering basis with primary users. This ideabehind is gaining traction with regulatory bodies and evidenced by the recent ruling of the FCC to open up a $700 \mathrm{MHz}$ bandfor the use of a dynamic basis.

5) A successful cognitive radio network (CRN) needs a fast and reliable spectrum sensing scheme to activate the secondary users to fulfil the available channels. The authors first revisit conventional models for channel availability (the random independent identical distribution models) and introduce a correlated Markov model which is proposed for scenarios with memory. the performance of random and serial search schemes for the above occupancy models in terms of the mean number of steps. then presented for different sensing schemes under both random and correlated channel model. The authors have to indicate the key which shows overall mean time to detect a free channel. It is function of bothmean number of steps and the sensing time per step. Reduced sensing duration in each step leads to lower detection probability thereby increasing the average number of search steps required. This suggests, there exists an optimal sensing duration that minimises the overall mean detection time. This is analytically investigated)and verified by simulation results under various SNR environments.

\section{RELATION TO EXISTING WORK AND FORMULATION}

\section{A. Channel Search}

There are two types of channel search i.e. random search and serial search. Assuming band to be searched is composed of $\mathrm{M}$ contagious channels having each of bandwidth. Considering number of unused channels is $\mathrm{L}$ out of $\mathrm{M}(\mathrm{L}<<\mathrm{M})$. Thus the probability of any channel being free can be denoted as $\lambda=\mathrm{L} / \mathrm{M}$ independent of all others. As shown in image; average no of search steps to detect an available channel for both serial \& random search for small $\mathrm{L} / \mathrm{M}$ is given by

$$
\overline{\mathrm{S}_{\mathrm{det}}}=\frac{1}{\mathrm{P}_{\mathrm{d}} \lambda}
$$

where "Pd is the detection probability of a free channel", i.e. the product $\operatorname{Pd} \lambda$ is the aggregate probabilityo a channel is actually free.

\section{B. ENERGY DETECTOR}

The given equation shows a binary hypothesis test for spectrum sensing of primary occupation,

$$
\begin{gathered}
\mathrm{H}_{0}: \mathrm{R}_{\mathrm{d}}=\mathrm{Z}_{\mathrm{s}} \\
\mathrm{H}_{1}: \mathrm{R}_{\mathrm{d}}=\mathrm{h}_{\mathrm{ps}} \mathrm{S}_{\mathrm{p}}+\mathrm{Z}_{\mathrm{s}}
\end{gathered}
$$

Where,

$\mathrm{Rd}=$ received signal at the detector of the secondary user,

H0 $($ hypothesis H0) = channel idle,

H1 (hypothesis H1) =channel busy,

$\mathrm{Sp}=$ Transmitted Power from Primary user.

Zs=Additive Noise for Primary users. 
The channel status is determined based on energy detection with $\mathrm{N}$ number of samples. Thus, the sensing duration is: $T_{i}=N / B_{c}$ and the test statistic for the energy detector is given by:

$$
\mathrm{Y}=\frac{1}{\mathrm{~N}} \sum_{\mathrm{i}=2}^{\mathrm{N}}\left|\mathrm{R}_{\mathrm{d}}(\mathrm{i})\right|^{2}
$$

Where, $\mathrm{Bc}$ is Bandwidth \& $\mathrm{N}$ is no. of samples.

We Can show the event "the secondary user claims that the channel is idle" as D0 and the event "the secondary user claims that the channel is busy" as D1. We then indicate the probability of successful detection of an available channel (hypothesis H0) as Pd and corresponding false alarm probability Pfa.Therefore we have

$P_{r}\left(D_{0} / H_{1}\right)=P_{f a}$

$$
\mathrm{P}_{\mathrm{r}}\left(\mathrm{D}_{0} / \mathrm{H}_{0}\right)=\mathrm{P}_{\mathrm{d}}
$$$$
P_{r}\left(D_{1} / H_{0}\right)=1-P_{d}
$$

$P_{r}\left(D_{1} / H_{1}\right)=1-P_{f a}$

For a given sensing threshold $\mathrm{x}, \mathrm{Pd}$ and $\mathrm{Pfa}$ are given by:

$$
\begin{gathered}
P_{d}(x, N)=P_{r}\left(Y<x \mid H_{0}\right) \\
=1-Q\left(\frac{x-\sigma^{2}}{\sigma_{n}^{2} \sqrt{2}} \sqrt{N}\right) \\
P_{f a}(x, N)=P_{r}\left(Y<x \mid H_{1}\right) \\
=1-Q\left(\frac{x-(\gamma+1) \sigma^{2}}{\sigma_{n}^{2}(\gamma+1) \sqrt{2}} \sqrt{N}\right)
\end{gathered}
$$

Where, $\lambda$ is the per-sample signal-to-noise ratio (SNR) at the energy detector of the secondary user and the Q-Function is:

$$
Q(x)=\frac{1}{\sqrt{2}} \int_{x}^{\infty} e^{t^{2} / 2} d t
$$

\section{IV .OPTIMIZATION APPROACH}

\section{A. Minimizing Mean Detection Time}

We have to minimize the average time to detect an available i.e. unused by primary users channel by secondary users as a function of two parameters of the energy detector. The threshold and the integration time over multiple channels involves two types of components: a) channel switching time (Ts) largely determined by the settling time of the phase locked loop (PLL) used in the receiver circuitry and $b$ ) integration time.

To arriveTi needed by the energy detector at a reliable detectionthis in turn is determined by the sensing bandwidth and the number of sensing samples. Thus, the net mean time to detect an available channel is given by

$$
\begin{aligned}
& \overline{T_{\text {det }}}=\overline{S_{\text {det }}}\left(T_{S+} T_{i}\right), \\
= & \frac{1}{P_{d} \lambda}\left(T_{s}+\frac{N}{B_{c}}\right)
\end{aligned}
$$

Where, Ts is channel switching time \& $\mathrm{Ti}$ is integration time and $\mathrm{Bc}$ is Sensing Bandwidth \& $\mathrm{N}$ is No. of sensing samples.
Therefore, the joint optimization problem is mathematically formulated as below:

$$
\min _{x, N} \overline{T_{\text {det }}}=\frac{1}{P_{d} \lambda}\left(T_{s}+\frac{N}{B_{c}}\right)
$$

Subject to

$$
\text { 1) } P_{d}(x, N) \geq \alpha
$$

2) $P_{f a}(x, N) \leq \beta$.

\section{B. Aggregate OPPORTUNistic ThroughPUT}

Minimizing the mean time to detect thespectral hole is an appropriate physical layer metric for optimization of a cognitiveNetwork. However, this ignores the impact of incorrect link layer decision for example, if there is a for a busy channel the false alarm, the primary and the secondary users will simultaneously transmit and cochannel interference will significantly suddenly decreases the throughput from both primary and secondary systems. Therefore, it is correct to use a MAC layer metric notably the aggregate opportunistic throughput of both primary and secondary users, so as to uncover newer system trade off not captured by the earlier method.

We consider that secondary users were periodically sense the spectrum by the frame. The duration of a frame is fixed length $T$, and thus the average transmission time for a secondary user is $\mathrm{T}-\mathrm{Tdet}$ per frame.Further a primary user uses whole frame duration to emit its signal when occupying the certain channel.We assume that the presence of a central controller which searches for available channel and then assign any such channels to the secondary user.We use the term ' $\mathrm{TH}$ ' to denote the opportunistic throughput per frame per channel.Thesearch scheme will ultimately find a channel for the secondary user, either in hypothesisH0 (channel vacant) or $\mathrm{H} 1$ (channel occupied).The search time of the wasted chances due to the miss detection probability is correlated to the calculating the average detection time. To Increase the average aggregate opportunistic throughput, we can calculate the problem as below:

$$
\min _{x, N} \overline{T H}
$$

Subject to 1) $P_{d}(x, N) \geq \alpha$;

2) $P_{f a}(x, N) \leq \beta$.

\section{PROPOSED SYSTEM TO IMPLEMENT}

Overview of the system algorithm described in following figure. RTL-SDR receives the FM signal as the input signal. This input signal given to MATLAB through controller. Controller convert the analog input signal to digital signal and transmit it tothe MATLAB.

MATLAB will analyze the input signal and calculate its respective parameter to find out $\mathrm{PU}$ in signal. If the primary user present in the signal then it again sense the spectrum till spectrum is vacant. If the PU is absent then it provide results that spectrum is vacant and other signal can be used for communication.

The proposed system consists of hardware modules: 


\section{RTL MODULE \\ 2. MATLAB \\ 3. ARM 7 \\ 4. FM Transmitter}

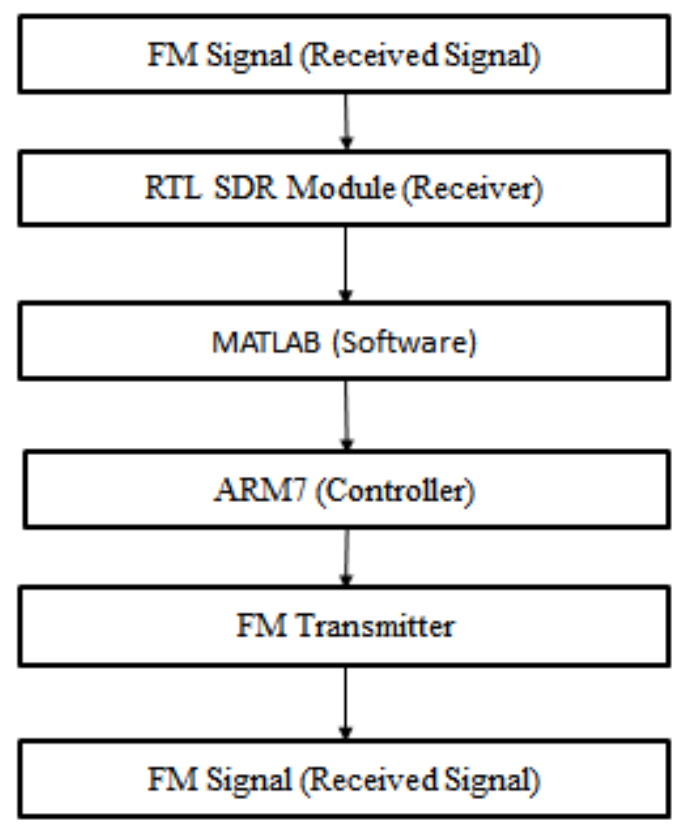

Fig. 1. Block Diagram for Proposed System to Implement

\section{SUMMERY}

In this paper Spectrum sensing optimization approach for cognitive radio network is proposed to minimize the average detection time of an available channel. Joint optimization of the sensing threshold and the number of sensing samples in the energy detector is proposed for the same. From numerical observations, we can say that proposed joint optimization can significantly increase in the average aggregate opportunistic throughput and decrease the mean time to detect spectrum hole of both primary and secondary users.

\section{REFERENCES}

[1] N .Monvkee"Qualifying the availability of Television White Spaces for Cognitive Radio Operation in the UK," ICC'09,pp 1-5 June 2008.

[2] J. Mitola III, Cognitive radio- An Integrated Agent architecture for Software Defined Radio Thesis, Swedish Royal Institute of Technology, 2000.

[3] Y.C. Liang, Y. Zeng, E. C. Y. Peh, and A.T. Hoang, "Sensing throughout Trade-offfor cognitive radio networks," IEEE Wireless Communication, Vol. 7, no. 4, pp. 1326-1337, Apr 2008.

[4] Z.QuanS.Cui, A. H. Sayed, and H. V. Poor"For Optimal andjoint detection for spectrum sensing in cognitive radio networks," IEEE Transactions onSignal Processing, vol57, no. 3, pp. 1128-1140, Mar, 2009.

[5] L. Luo, N. M. Neihart, S. Roy, and D. J Allstot, "A Two-stage sensing technique for Dynamic spectrum access," IEEE transfers on Wireless communication, Vol. 8, No. 6, pp. 3028-3037, June 2009.

[6] L. Luo and S. Roy, Analysis of search schemes in cognitive radio," SECON'07, pp. 647-654, June 2007.
[7] Ling Luo and Sumit Roy, "Efficient Spectrum Sensing for the cognitive Radio Networks via Joint Optimization of SensingThreshold and Duration " IEEE Transactions on Communications vol. 60 No. 10, Oct 2012.

[8] J. Gorski, F. Pfeuffer, and K. Klamroth, "Biconvex sets and optimization with biconvex functions: A survey and extensions," Math. Meth. OfReservation, Vol. 66, No. 3, pp. 373-407, Dec 2007.

[9] C.A. floudsand V. Visweswaran, "A global optimization algorithm (GOP) for certain classes of a non-convex NLPSI. Theory", Computer Chem. Eng., Vol. 14, No. 12, July 1990.

[10] L. Luo and S. Roy "Modeling and analysis of detection time tradeoffs for channel searching a cognitiveradio networks" to be published.

\section{BIOGRAPHIES}

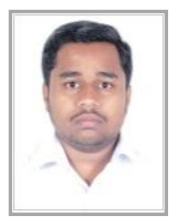

Mr. Vinayak R. Barbole received BE (E\&TC) degree from Savitribai Phule Pune University,Pune. He is now pursuing for ME (VLSI \& Embedded) from Savitribai Phule Pune University, Pune Maharashtra, India.

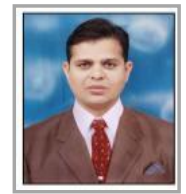

Prof. Anant R. More is currently working as Asst. Professor in Sinhgad Institutes and is a part time Ph.D. Scholar of K.L. University. His fields of interest in E\&TC engineering are Wireless Communication and Digital VLSI Design.

Prof. M.S.G. Prasad is currently working as Asst. Professor in Electronics and communication Department, KKR Institute of Technology \& Sciences, Guntur, India. His fields of interest in E\&TC engineering are Wireless Communication and Digital VLSI Design. 